\title{
Surface Alignment of 3D Spherical Harmonic Models: Application to Cardiac MRI Analysis
}

\author{
Heng Huang ${ }^{1}$, Li Shen ${ }^{3}$, Rong Zhang ${ }^{1}$, Fillia Makedon ${ }^{1}$, \\ Bruce Hettleman ${ }^{2}$, and Justin Pearlman ${ }^{1,2}$ \\ 1 Department of Computer Science, Dartmouth College, Hanover, NH 03755 \\ 2 Department of Cardiology, Dartmouth Medical School, Lebanon, NH 03756 \\ ${ }^{3}$ Computer and Information Science Department, Univ. of Mass. Dartmouth, MA 02747 \\ hh@cs. dartmouth. edu
}

\begin{abstract}
The spherical harmonic (SPHARM) description is a powerful surface modeling technique that can model arbitrarily shaped but simply connected 3D objects and has been used in many applications in medical imaging. Previous SPHARM techniques use the first order ellipsoid for establishing surface correspondence and aligning objects. However, this first order information may not be sufficient in many cases; a more general method for establishing surface correspondence would be to minimize the mean squared distance between two corresponding surfaces. In this paper, a new surface matching algorithm is proposed for 3D SPHARM models to achieve this goal. This algorithm employs a useful rotational property of spherical harmonic basis functions for a fast implementation. Applications of medical image analysis (e.g., spatio-temporal modeling of heart shape changes) are used to demonstrate this approach. Theoretical proofs and experimental results show that our approach is an accurate and flexible surface correspondence alignment method.
\end{abstract}

\section{Introduction}

Surface representation and shape modeling play increasingly prominent roles in many computer vision and image processing applications. Medical image analysis is one of the most important applications. Many techniques have been developed for modeling and inspecting anatomic structures in the diagnosis and treatment of disease. The spherical harmonics approach has been used for the representation of shapes in many types of biomedical image data to help perform functional information analysis or classify different pathological symptoms.

Many spherical harmonic based shape descriptions have been developed for medical image analysis. Chen et al. [1] use this method to model and analyze left ventricular shape and motion. Matheny et al. [2] and Burel et al. [3] use 3D and 4D surface harmonics to reconstruct rigid and nonrigid shapes. Since they start from an initial radial surface function $r(\theta, \phi)$, their method is capable of representing only star-shaped or convex objects without holes. Brechbühler et al. [4] present the SPHARM description that is an extended spherical harmonic method for modeling any simply connected 3D object. The object surface is represented as $\mathbf{v}(\theta, \phi)=(x(\theta, \phi), y(\theta, \phi), z(\theta, \phi))^{T}$ and spherical harmonics expansion is used for all three coordinates. Gerig and Styner have 
applied SPHARM in many medical imaging applications (e.g., shape analysis of brain structures [567]). It has also been used for shape modeling and functional analysis for cardiac MRI [8].

In order to compare different SPHARM models, a shape registration step is often necessary for aligning these models together and extracting their shape descriptors (i.e., excluding translation, rotation, and scaling). Like shape registration using ICP [9], two important substeps are involved in aligning SPHARM models: (1) creating surface correspondence, and (2) minimizing the distance between the corresponding surface parts. Once the surface correspondence is established, the distance minimization becomes relatively easy. Thus, the focus of this paper is on creating surface correspondence for two 3D SPHARM models.

Previous studies [56] used the first order ellipsoid for shape registration. The parameter net on this ellipsoid is rotated to a canonical position such that the north pole is at one end of the longest main axis, and the crossing point of the zero meridian and the equator is at one end of the shortest main axis. The aligned parameter space creates surface correspondence between two models: two points with the same parameter pair $(\theta, \phi)$ on two surfaces are defined to be a corresponding pair. This alignment technique works only if the first order ellipsoid is a real ellipsoid, as in the case of hippocampal data [6], but not if it is an ellipsoid of revolution or a sphere. There are also other cases in which first order ellipsoid alignment may not work. One example is given in Fig. 1 for the heart ventricle case.

In this paper, instead of aligning the first order ellipsoid, we employ a more general metric for establishing surface correspondence: minimizing the mean squared distance between two SPHARM surfaces. A fast surface alignment algorithm is proposed to achieve this. Based on the rotational properties of harmonics analysis, we prove that a new set of SPHARM coefficients after a rotated parametrization can be directly generated from the original set. Thus we can easily obtain a new SPHARM model for a re-parameterized object by rotating its parametrization along the surface. This process is faster than a standard recalculation (e.g., solving a linear equation) of SPHARM coefficients for a re-parameterized object. This work is motivated by the need for better shape modeling and analysis in current medical applications. Some of these applications are used to demonstrate our algorithm in this paper.

\section{Methods}

\subsection{Surface Description Using SPHARM}

The SPHARM technique [4] can be used to model arbitrarily shaped, simply connected 3D objects. The object surface is represented by using spherical harmonics expansion for all three coordinates,

$$
\mathbf{v}(\theta, \phi)=(x(\theta, \phi), y(\theta, \phi), z(\theta, \phi))^{T}=\sum_{l=0}^{\infty} \sum_{m=-l}^{l} \mathbf{c}_{l}^{m} Y_{l}^{m}(\theta, \phi) .
$$

The coefficients $\mathbf{c}_{l}^{m}=\left(c_{l x}^{m}, c_{l y}^{m}, c_{l z}^{m}\right)^{T}$ are $3 \mathrm{D}$ vectors. Their components, $c_{l x}^{m}, c_{l y}^{m}$, and $c_{l z}^{m}$ are usually complex numbers. The coefficients up to a user-desired degree can be 
estimated by solving a set of linear equations in a least square fashion. The object surface can be reconstructed using these coefficients, and using more coefficients leads to a more detailed reconstruction. Thus, a set of coefficients actually form an object surface description.

\subsection{Fast Rotation Theorem for Spherical Harmonic Parametrization}

Theorem (Parametrization Rotation). The parametrization spatial rotation on the surface can be decomposed into three rotations of mapping parameter meshes onto the $x$-sphere, $y$-sphere, and $z$-sphere. Let $\mathbf{v}(\theta, \phi)=\sum_{l=0}^{\infty} \sum_{m=-l}^{l} \mathbf{c}_{l}^{m} Y_{l}^{m}(\theta, \phi)$ be a SPHARM parametric surface. After rotating the parameter net on the surface in Euler angles $(\alpha, \beta, \gamma)$, the new coefficients $c_{l}^{m}(\alpha \beta \gamma)$ is

$$
c_{l}^{m}(\alpha \beta \gamma)=\sum_{m^{\prime}=-l}^{l} c_{l}^{m^{\prime}} D_{m m^{\prime}}^{l}(\alpha \beta \gamma)
$$

Proof. According to Euler's rotation theorem, any rotation of the coordinate system $\left(e_{1}, e_{2}, e_{3}\right)$ can be decomposed into three elementary rotations $R(\alpha, \beta, \gamma)$. The $S O(3)$ harmonics provide the tool to express the rotated version of a function on the sphere extended by spherical harmonics [10]. The effect of such a rotation on the spherical harmonic basis functions is [11]

$$
R_{Z Y Z}(\alpha \beta \gamma) Y_{l}^{m}(\theta, \phi)=\sum_{m^{\prime}=-l}^{l} Y_{l}^{m^{\prime}}(\theta, \phi) D_{m^{\prime} m}^{l}(\alpha \beta \gamma),
$$

where $R_{Z Y Z}(\alpha \beta \gamma)$ represents the rotation operator dependent on the Euler angles; the rotation matrices $D_{m^{\prime} m}^{l}(\alpha \beta \gamma)$ (also called the $S O(3)$ matrix elements) are calculated by

$$
D_{m^{\prime} m}^{l}(\alpha \beta \gamma)=e^{-i m^{\prime} \alpha} d_{m^{\prime} m}^{l}(\beta) e^{-i m \gamma},
$$

where

$$
\begin{aligned}
d_{m^{\prime} m}^{l}(\beta)= & \sum_{t=\max \left(0, m-m^{\prime}\right)}^{\min \left(l+m, l-m^{\prime}\right)}(-1)^{t} \times \frac{\sqrt{(l+m) !(l-m !)\left(l+m^{\prime}\right) !\left(l-m^{\prime}\right) !}}{(l+m-t) !\left(l-m^{\prime}-t\right) !\left(t+m^{\prime}-m\right) ! t !} \\
& \times\left(\cos \frac{\beta}{2}\right)^{\left(2 l+m-m^{\prime}-2 t\right)}\left(\sin \frac{\beta}{2}\right)^{\left(2 t+m^{\prime}-m\right)} .
\end{aligned}
$$

Since we employ the SPHARM surface modeling technique, the surface coordinate information of a 3D object is coded onto three unit spheres: an $x$-sphere, a $y$-sphere, and a $z$-sphere. These three spherical functions are expanded using spherical harmonics and represented by $f(\theta, \phi)(f \in\{x, y, z\})$. We denote $f^{\prime}(\theta, \phi)$ as the new function after applying a rotation operator $R_{Z Y Z}(\alpha \beta \gamma)$ to $f(\theta, \phi)$ on the $f$-sphere:

$$
f^{\prime}(\theta, \phi)=R_{Z Y Z}(\alpha \beta \gamma) f(\theta, \phi)
$$


thus

$$
\mathbf{v}^{\prime}(\theta, \phi)=R_{o b j}(\alpha \beta \gamma)(x(\theta, \phi), y(\theta, \phi), z(\theta, \phi))^{T}
$$

where $R_{o b j}(\alpha \beta \gamma)=\left[R_{Z Y Z}(\alpha \beta \gamma) 00 ; 0 R_{Z Y Z}(\alpha \beta \gamma) 0 ; 00 R_{Z Y Z}(\alpha \beta \gamma)\right]$.

Then $\mathbf{v}^{\prime}(\theta, \phi)=R_{o b j}(\alpha \beta \gamma) \mathbf{v}(\theta, \phi)$ represents the new parametrization on the surface, which can be generated by rotating the original parametrization along the object's surface about Euler angles $(\alpha, \beta, \gamma)$. In other word, the result of applying the rotation matrix $R_{Z Y Z}(\alpha \beta \gamma)$ on the mapping meshes of $x, y, z$-sphere is to rotate the parameter mesh on the object's surface at the same orientation. Because of the distortions introduced by spherical parameterization, the result of rotation is not identical to the result of applying Euler angles on the sphere, but both will have nearly the same orientation. Thus we only use $R_{o b j}$, which we refer to as the parametric rotation matrix, for rotating the parameter mesh along the surface of an object. Substituting Eq. (1) and Eq. (3) into Eq. (4) gives

$$
\begin{aligned}
& \sum_{l=0}^{L} \sum_{m=-l}^{l} c_{l f}^{m}(\alpha \beta \gamma) Y_{l}^{m}(\theta, \phi)=f^{\prime}(\theta, \phi)=R_{Z Y Z}(\alpha \beta \gamma) \sum_{l^{\prime}=0}^{L} \sum_{m^{\prime}=-l^{\prime}}^{l^{\prime}} c_{l^{\prime} f}^{m^{\prime}} Y_{l^{\prime}}^{m^{\prime}}(\theta, \phi)= \\
& \sum_{l^{\prime}=0}^{L} \sum_{m^{\prime}=-l^{\prime}}^{l^{\prime}} c_{l^{\prime} f}^{m^{\prime}} R_{Z Y Z}(\alpha \beta \gamma) Y_{l^{\prime}}^{m^{\prime}}(\theta, \phi)=\sum_{l^{\prime}=0}^{L} \sum_{m^{\prime}=-l^{\prime}}^{l^{\prime}} c_{l^{\prime} f}^{m^{\prime}} \sum_{n=-l^{\prime}}^{l^{\prime}} Y_{l^{\prime}}^{n}(\theta, \phi) D_{n m^{\prime}}^{l^{\prime}}(\alpha \beta \gamma)
\end{aligned}
$$

and multiplying $\bar{Y}_{k}^{j}(\theta, \phi)$ on both sides (adjusting the $k$ from 0 to $L$ and $j$ from $-k$ to $k$ ) and integrating on the sphere. Since all Kronecker delta values are zero except at $k=l=l^{\prime}$ and $j=m=n$, we get the following:

$$
c_{l f}^{m}(\alpha \beta \gamma)=\sum_{m^{\prime}=-l^{\prime}}^{l^{\prime}} c_{l^{\prime} f}^{m^{\prime}} D_{m m^{\prime}}^{l^{\prime}}(\alpha \beta \gamma)=\sum_{m^{\prime}=-l}^{l} c_{l f}^{m^{\prime}} D_{m m^{\prime}}^{l}(\alpha \beta \gamma) .
$$

According to the above derivation, the harmonics expansion coefficients transform among themselves during rotation. Each new spherical harmonic coefficient $c_{l f}^{m}(\alpha \beta \gamma)$ after applying a rotated function $R_{Z Y Z}(\alpha \beta \gamma)$ is a linear combination of the coefficients $c_{l f}^{m}$ of the original function $f(\theta, \phi)(f \in\{x, y, z\})$. We can use this property to calculate the new SPHARM model $\mathbf{v}^{\prime}(\theta, \phi)$ for the object surface after a rotated parametrization, and we only need the old coefficients $\left\{c_{l x}^{m}, c_{l y}^{m}, c_{l z}^{m}\right\}$ and rotation matrices $D_{m m^{\prime}}^{l}(\alpha \beta \gamma)$.

\subsection{Surface Correspondence Difference Measure}

The surface correspondence alignment problem is generally formulated in terms of the optimal parameters, such as $(\alpha, \beta, \gamma)$, that minimize some surface distance function. In this paper, we adopt the Euclidean distance as the distance function between surfaces. Formally, for two surfaces given by $\mathbf{v}_{1}(s)$ and $\mathbf{v}_{2}(s)$, their distance $D\left(\mathbf{v}_{1}, \mathbf{v}_{2}\right)$ is defined as [6]

$$
D\left(\mathbf{v}_{1}, \mathbf{v}_{2}\right)=\left(\sum_{f \in\{x, y, z\}} \sum_{l=0}^{L} \sum_{m=-l}^{l}\left(c_{l f_{1}}^{m}-c_{l f_{2}}^{m}\right)^{2}\right)^{1 / 2} .
$$




\subsection{Fast Surface Alignment Algorithm}

We use a sampling-based search algorithm, which fixes one parameter mesh and rotates the other to carry out a greedy search on its surface with a small step size, to align the surface correspondence by minimizing the surface distance defined in Eq. (7). First, we uniformly distribute sample points on the surface as the new north poles locations, and the new SPHARM coefficients can be calculated by using Eq. (2) with new Euler angles set $(\alpha, \beta, \gamma)$. For each candidate north pole, the parameter mesh is counterclockwise rotated along the new north-south aixes (the rotation angle $\omega$ ranges from 0 to $2 \pi$ ). In order to calculate the coefficients of the new rotated parameter mesh using Eq. (2), we must transform the rotation angle $\omega$ into the Euler angles $(\alpha, \beta, \gamma)$.

The original north and south poles of a surface's parameter mesh are mapped onto the axis $e_{3}=\left(\begin{array}{lll}0 & 0 & 1\end{array}\right)$ and $-e_{3}=\left(\begin{array}{lll}0 & 0 & -1\end{array}\right)$ in the $x$-, $y$-, and $z$-sphere. After rotation using Euler angles $\left(\alpha_{p}, \beta_{p}, \gamma_{p}\right)$, the north and south pole coordinates switch from $v(\theta, \phi)$ to $v^{\prime}(\theta, \phi)=R_{o b j}\left(\alpha_{p} \beta_{p} \gamma_{p}\right) v(\theta, \phi),(\theta=0$ or $\pi)$. Simultaneously the axis $e_{3}$ in the coordinate systems of the three mapping spheres is changed to $\hat{e}_{3}=$ $R_{Z Y Z}\left(\alpha_{p} \beta_{p} \gamma_{p}\right)\left(\begin{array}{lll}0 & 0 & 1\end{array}\right)^{T}$. Because $\hat{e}_{3}$ also contains the origin and has unit length direction, we apply the Rodrigues' rotation formula [13] for computing the rotation matrix $R_{\hat{e}_{3}} \in S O(3)$ corresponding to a rotation by an angle $\omega$ about the fixed axis $\hat{e}_{3}$

$$
R_{\hat{e}_{3}}(\omega)=I+S \sin \theta+S^{2}(1-\cos \theta), \quad S=\left[\begin{array}{ccc}
0 & -\hat{e}_{3 x} & \hat{e}_{3 y} \\
\hat{e}_{3 z} & 0 & -\hat{e}_{3 x} \\
-\hat{e}_{3 y} & \hat{e}_{3 x} & 0
\end{array}\right],
$$

where $I$ is the identity matrix. We can obtain the Euler angles $(\alpha, \beta, \gamma)$ by solving the equation $R_{Z Y Z}(\alpha \beta \gamma)=R_{\hat{e}_{3}}(\omega)$. These Euler angles can then be used to calculate the coefficients of new parameter mesh using Eq. (2).

In the second step, we use the BFGS algorithm [14] to locally minimize Eq. (7) starting from the result of the first step. Because the result of the first step is already close to the target, this step generally needs only a few iterations. Although the dimension of the Jacobian matrix is large, the matrix is quite sparse. The computational time of this step is very low.

\section{Experiments and Discussions in Medical Image Analysis}

The fast alignment algorithm for surface correspondence described above was used for shape analysis in selected medical image analysis applications. Based on segmented MRI data of heart, we use the SPHARM method to do surface reconstruction and apply the surface alignment algorithm presented in this paper to determine a correspondence between shapes. This aligned correspondence allows researchers to access more functional details.

\subsection{Comparison of Methods}

In previous shape analysis study using the SPHARM description [5], researchers choose to use the three major axis of the first order ellipsoid (which is computed from the 


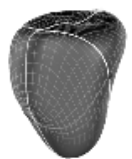

(a)

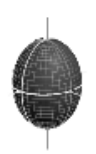

(f)

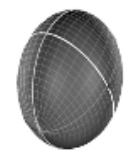

(b)

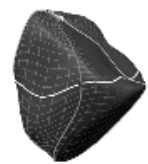

(g)

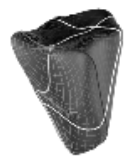

(c)

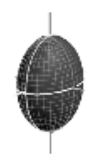

(h)

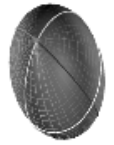

(d)

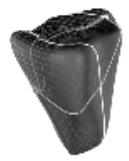

(i)

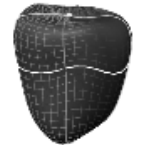

(e)

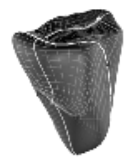

(j)

Fig. 1. Comparison of methods: (a) shows the reconstructed SPHARM surface of left ventricle, (b) is the first order ellipsoid of surface (a); (c) shows the reconstructed SPHARM surface of right ventricle, (d) is the first order ellipsoid of surface (c). By using the previous method, the first order ellipsoids and parametrizations are rotated to the positions in (f) and (h), and the SPHARM surfaces and parametrizations are rotated as (e) and (g). By using our algorithm, (i) shows the result of poles alignment. North and south poles are aligned close to the poles of (a). And the parameter mesh is rotated along the north pole. After using the BFGS algorithm [14] in the second step, the last alignment result is shown in ( $\mathrm{j}$ ).

first order SPHARM coefficients) as the intrinsic coordinate system. Parametrization is rotated in the parameter space for normalization so that three main ridges of the first order ellipsoid are moved to the equator [5|6]. Their method works well if two or more objects have a similar orientation (e.g., aligning hippocampal shapes). However this method may not work in some cases.

Fig.1. (a) and Fig.1 (c) show the reconstructed surface of two ventricles of the heart (left ventricle and right ventricle). We separate the parametrization on the surface into eight regions using five lines $(\theta=\pi / 2$ in middle line, in north pole $\theta=0$, in south pole $\theta=\pi$, the other four lines separately represent $\phi=0, \pi / 2, \pi, 3 \pi / 2,2 \pi$ ). The correspondences between surfaces in Fig. 1.(a) and Fig. 1.(c) are unordered as the visualizations. Fig. 1.(b) and Fig. 1.(d) show their first order ellipsoids. By using the previous method, the first order ellipsoids and parametrizations are rotated to the positions in Fig. 1.(f) and Fig. 1.(h). Three main directions of the ellipsoids are moved to the equator. The surface correspondence is created when the first order ellipsoid is aligned. As the result, the SPHARM surfaces and parametrizations should be rotated as Fig. 1.(e) and Fig.1 (g). A limitation of this approach is that it may not represent the real surface correspondence between two surfaces. The reason for this is that the left ventricle and right ventricle have two very different orientations of their first order ellipsoid that are obvious in Fig.1.(b) and Fig.1.(d). Thus, although the first order ellipsoids are rotated to the normalized positions, the surfaces are rotated to the opposite orientations.

Our new alignment algorithm produces a correct alignment in these cases, because it is a general surface alignment method that does not depend on any orientation information. Fig. 1.(i) and Fig. 11(j) show the results generated by our algorithm. Fig. 1. (a) is the fixed surface and the parametrization in Fig. 1.(c) is rotated to Fig. 1.(j).

The effectiveness of our algorithm can also be demonstrated by computing the surface correspondence distance defined in Eq. (7). The surface correspondence distance 
between surfaces in Fig.1. (e) and Fig.11. (g) is 258.6536mm, but the surface correspondence distance between surfaces in Fig.1.(a) and Fig. 1.(j) is 62.4798m . Our surface alignment algorithm derives a better result.

\subsection{Alignment for Temporal Heart Sequences}

This new surface alignment algorithm also provides a promising method for studying spatio-temporal structures. In the previous research [15], surface tracking techniques (tracking points on 3D shape using 2D images) are used to create temporal sequence descriptions for points on the left ventricle inner surface through each heart cycle. Such temporal sequence descriptions can quantify the ventricular mechanical asynchrony or synchrony, which has important diagnostic and prognostic values, and can help determine optimal treatment in heart failures where a heart has a highly asynchronous contraction. Because the points are tracked on 2D images and mapped to a 3D surface, this method can only describe the heart contraction and dilation along the plane direction, and is not accurate for the perpendicular direction.

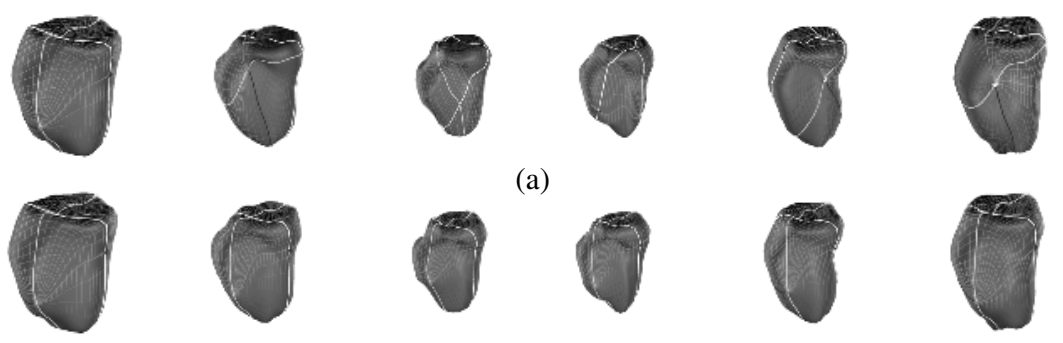

(b)

Fig. 2. (a) shows a shape sequence of a left ventricular inner surface during one heart cycle before surface alignment. The shape sequence in (b) is the result after surface alignment.

Combining the SPHARM description and our surface alignment methods offers a set of spatio-temporal surface correspondences for medical image analysis research. Our new algorithm generates more reasonable surface correspondences for the left ventricle sequence, and these surface correspondences describe the heart contraction and dilation in every direction of 3D space. Based on this new model, more valuable diagnostic and prognostic information can be derived for helping make clinical determinations.

Fig. 2. (a) is a shape sequence of a left ventricular inner surface during one heart cycle. Before surface alignment, the parametrization of every surface is unordered. The shape sequence in Fig. 2(b) is the result after surface alignment. During the alignment procedure, every shape is aligned with its anterior shape.

\section{Conclusions}

This paper addresses the problem of finding surface correspondences between SPHARM parametric surfaces. We propose a theorem based on the SPHARM rotational property 
to easily calculate SPHARM coefficients of the new parameter mesh along the surface. The distance between surfaces is defined as the objective function. Its efficacy is demonstrated in experiments based on several medical research problems, where we observe a significant improvement in robustness relative to existing shape modeling and analysis techniques.

There are several future directions. 1) The current algorithm can be integrated into an ICP-like framework for registration of 3D parametric surfaces. After finding the corresponding points on the surfaces, we can use an ICP-like algorithm to minimize the value of the correspondence. 2) Considerable research has focused on generating an equal area parametric mesh for a given surface. Our alignment algorithm can be combined with such parametrization methods to produce a better surface correspondence. These results are very useful for the shape and functional analysis in medical imaging.

\section{References}

1. Chen Chang Wen, Huang, T.S., Arrott, M.: Modeling, analysis, and visualization of left ventricle shape and motion by hierarchical decomposition. IEEE PAMI 16(4) (1994) 342-356.

2. Matheny, A., Goldgof, D.B.: The use of three- and four-dimensional surface harmonics for rigid and nonrigid shape recovery and representation. IEEE PAMI 17(10) (1995) 967-981.

3. Burel, G., Hennocq, H.: Determination of the Orientation of 3D Objects Using Spherical Harmonics. Graphical Models and Image Processing 57(5) (1995) 400-408.

4. Brechbühler, Ch., Gerig, G., and Kübler, O.: Parametrization of closed surfaces for 3D shape description. Computer Vision and Image Understanding 61(2) (1995) 154-170.

5. Styner, M., Gerig, G.: Three-Dimensional Medial Shape Representation Incorporating Object Variability. Proc. IEEE Conf. Computer Vision and Pattern Recognition (2002) 651-656.

6. Gerig, G., Styner, M.: Shape versus Size: Improved Understanding of the Morphology of Brain Structures. International Conference on Medical Image Computing and Computer Assisted Intervention LNCS 2208 (2001) 24-32.

7. Styner, M., Lieberman, A.J., Pantazis, D., and Gerig, G.: Boundary and Medial Shape Analysis of the Hippocampus in Schizophrenia. Med. Im. Ana. MEDIA. 8(3) (2004) 197-203.

8. Huang, H., Shen, L., Ford, J., Makedon, F., Zhang, R., et al.: Functional analysis of cardiac MR images using SPHARM modeling. Proceedings of the SPIE 5747 (2005) 1384-1391.

9. Besl, P. J., McKay, N. D.: A method for registration of 3-D shapes. IEEE Trans. on Pattern Analysis and Machine Intelligence 14(2) (1992) 239-256.

10. Chirikjian, G.S., Kyatkin, A.B.: Engineering Applications of Noncommutative Harmonic Analysis: With Emphasis on Rotation and Motion Groups. CRC Press (2000).

11. Biedenharn, L.C., Louck, J.C.: Angular Momentum in Quantum Physics. Addison-Wesley: Reading MA (1981).

12. Ritchie, D.W., Kemp, G.J.L.: Fast computation, rotation, and comparison of low resolution spherical harmonic molecular surfaces. J. Comp. Chem. 20(4) (1999) 383-395.

13. http://mathworld.wolfram.com

14. Fletcher, R.: Practical Methods of Optimization. Princeton University Press, John Wiley and Sons, 2nd edition (1987).

15. Huang, H., Shen, L., Makedon, F., Zhang, S., Greenberg, M., Gao, L., Pearlman, J.: A clustering-based approach for prediction of cardiac resynchronization therapy. ACM Symposium on Applied Computing. (2005) 260-266. 